Check for updates

Cite this: RSC Adv., 2019, 9, 37573

Received 14th October 2019

Accepted 13th November 2019

DOI: $10.1039 / c 9 r a 08372 c$

rsc.li/rsc-advances

\section{Three-dimensional reduced graphene oxide aerogel stabilizes molybdenum trioxide with enhanced photocatalytic activity for dye degradation $\uparrow$}

\author{
Ting Wu,,$^{\mathrm{a}}$ Bo Zhang,,$^{* \mathrm{~b}}$ Zhimin $\mathrm{Wu},{ }^{* \mathrm{c}}$ Jinglin Zhang, ${ }^{\mathrm{a}}$ Huidi Liu, ${ }^{\mathrm{e}}$ Shaobin $\mathrm{Yu},{ }^{\mathrm{d}}$ \\ Zhihao Huang ${ }^{a}$ and Xiang Cai (D) *a
}

By using three-dimensional reduced graphene oxide $(\mathrm{rGO})$ aerogel as a carrier for molybdenum trioxide $\left(\mathrm{MoO}_{3}\right)$, a series of $\mathrm{rGO}-\mathrm{MoO}_{3}$ aerogels were synthesized by a self-assembly process. The results indicated that the as-prepared $\mathrm{rGO}-\mathrm{MoO}_{3}$ aerogel had very low density and good mechanical properties, and would not deform under more than 1000 times its own pressure. The $\mathrm{rGO}-\mathrm{MoO}_{3}$ aerogel showed more than $90 \%$ degradation efficiency for MB within 120 min. After six cycles of recycling, the degradation rate of $M B$ only decreased by $1.6 \%$. As supported by the electron paramagnetic resonance (EPR) measurements, the presence of the rGO aerogel enhanced electron conduction, prolonged carrier lifetime and inhibited electron and hole recombination, thus improving the photocatalytic efficiency of composite aerogel. Besides, the hydroxyl radical $\left(\mathrm{OH}^{*}\right)$ and radical anion $\left(\cdot \mathrm{O}_{2}{ }^{-}\right)$played an important role in the photodegradation of the dye. The outstanding adsorption and photocatalytic degradation performance of the $\mathrm{rGO}-\mathrm{MoO}_{3}$ aerogel was attributed to its unique physical properties, such as high porosity, simple recycling process, high hydrophobicity, low density and excellent mechanical stability. The findings presented herein indicated that the $\mathrm{rGO}-\mathrm{MoO}_{3}$ aerogel had good application potential, and could serve as a promising photocatalyst for the degradation of dyes in wastewater

\section{Introduction}

In human manufacturing activities, water resources will inevitably be polluted by organic matter, inorganic matter and microorganisms, ${ }^{\mathbf{1 , 2}}$ and the maintenance of clean water has become a serious global problem with the acceleration of industrialization, which is particularly prominent in developing

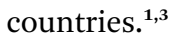

The waste of organic dyes from various industries is the largest component of organic pollutants in water. Around $10^{5}$ tons of various organic dyes are released into the environment

\footnotetext{
${ }^{a}$ Department of Light Chemical Engineering, Guangdong Polytechnic, Foshan 528041, P. R. China.E-mail: cecaixiang@163.com

${ }^{b}$ School of Metallurgical and Material Engineering, Hunan University of Technology, Zhuzhou 412007, P. R. China.E-mail: 13747@hut.edu.cn

${ }^{c}$ Human Resource Office, Guangdong Polytechnic, Foshan 528041, P. R. China. E-mail: carrybeyond@126.com

${ }^{d}$ The No.1 Surgery Department of No.5 People's Hospital of Foshan, Foshan 528211, P. R.China

${ }^{e}$ Scientific Research Office, Guangdong Polytechnic, Foshan 528041, P. R. China

$\dagger$ Electronic supplementary information (ESI) available. See DOI: 10.1039/c9ra08372c

\$ T. Wu and B. Zhang contributed equally to this article.
}

every year. ${ }^{4}$ The dosage of dyes in China accounts for $40-45 \%$ of the world's total. Due to the huge consumption of dyes, the situation of dye pollution in China is particularly severe. ${ }^{5}$ Organic dyes are very stable and difficult to decompose in aqueous solutions. Organic dyes discharged into rivers and lakes will cause serious water pollution, which will pose a great threat to the survival of aquatic organisms and human health. ${ }^{6}$ Methylene blue (MB) and methyl orange (MO) are considered to be the most common toxic dyes, causing skin diseases, genetic mutations and cancer to humans, animals and plants, etc. So, it is urgent to develop efficient and environmentally friendly technology to combat organic dye pollutants in water.,

At present, the main methods to remove organic pollutants in water are divided into physical method, chemical method and biological method. ${ }^{8}$ Physical methods mainly include membrane separation, filtration and evaporation. The chemical method mainly includes adsorption method, REDOX method and neutralization method. Biological methods mainly include activated sludge method and biological filtration method. Among these methods, adsorption method and photodegradation method are widely used in the purification of water pollution due to their convenience, high efficiency and economy, and they will not produce other harmful substances during the process of 
treatment and bring secondary pollution to the water body. ${ }^{\mathbf{9}, 10}$ Specially, photocatalytic technology is an effective means to convert solar energy into chemical energy to generate clean energy and degrade pollutants in the environment. ${ }^{9-12}$

Molybdenum trioxide $\left(\mathrm{MoO}_{3}\right)$ is an n-type semiconductor with good photochromic and electrochromic characteristics, which is suitable for various fields, including energy storage, gas sensing devices, catalytic applications and electrochemical devices. ${ }^{\mathbf{1 3 - 1 6}}$ In recent years, $\mathrm{MoO}_{3}$ has become a research object in the field of photocatalysis due to its large specific surface area and electron transmission along the axis, etc., but the photocatalytic degradation ability of $\mathrm{MoO}_{3}$ is limited due to its large band gap width and high photo-generated electron recombination rate. ${ }^{17-21}$ Graphene aerogels are characterized by large specific surface area, good hydrophilicity and effective improvement of photocatalytic property of material. ${ }^{22-26}$

In order to improve the photocatalytic degradation performance of $\mathrm{MoO}_{3}$, by loading $\mathrm{MoO}_{3}$ on reduced graphene oxide (rGO) aerogel, the rapid electron conduction could be achieved by virtue of the excellent electrical conductivity of rGO sheet layer to inhibit the combination of photo-generated electron and hole, thereby improving the photocatalytic efficiency.

In this study, MB and MO were selected as representative organic dyes, and the degradation effect of $\mathrm{rGO}-\mathrm{MoO}_{3}$ aerogel under simulated visible light on $\mathrm{MB}$ and $\mathrm{MO}$ in aqueous solution was investigated. Moreover, the degradation behavior of rGO- $\mathrm{MoO}_{3}$ aerogel was investigated from the aspects of reaction kinetics and photocatalytic degradation mechanism.

\section{Materials and methods}

\subsection{Materials}

Graphite powder (spectral pure) and all other analytical grade reagents (phosphorus pentoxide, concentrated sulfuric acid, potassium permanganate, hydrogen peroxide, sodium hydroxide, molybdenum pentachloride, anhydrous ethanol, ascorbic acid, sodium chloride, methylene blue and methyl orange) were purchased from Sinopharm Chemical Reagent Co., Ltd and used as received without further purification. All aqueous solutions used for the experiments were prepared with deionized water.

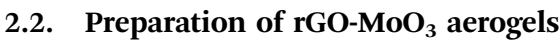

A typical preparation of $\mathrm{MoO}_{3}$ was described as follows: $0.325 \mathrm{~g}$ of $\mathrm{MoCl}_{5}$ was completely dissolved in the mixed solution of
$40 \mathrm{~mL}$ of deionized water and $10 \mathrm{~mL}$ of anhydrous ethanol. After continuous stirring for $1 \mathrm{~h}$, the $\mathrm{MoCl}_{5}$ solution was transferred to the polytetrafluoroethylene reactor and placed in the mufter furnace for reaction at $180{ }^{\circ} \mathrm{C}$ for $10 \mathrm{~h}$. After being cooled to room temperature in the air, the generated precipitation was centrifuged and separated, washed with anhydrous ethanol and deionized water for several times, and dried in an oven at $80{ }^{\circ} \mathrm{C}$ for $3 \mathrm{~h}$ to get impure $\mathrm{MoO}_{3}$. In order to completely convert $\mathrm{MoCl}_{5}$ into $\mathrm{MoO}_{3}$, the impure $\mathrm{MoO}_{3}$ powders were putted in a porcelain crucible, and continued to react at $450{ }^{\circ} \mathrm{C}$ for $1 \mathrm{~h}$ in muff furnace. After cooling to room temperature, the obtained powder was denoted as $\mathrm{MoO}_{3}$.

A typical preparation of $\mathrm{rGO}-\mathrm{MoO}_{3}$ aerogel was described as follows: graphene oxide was prepared through the modified Hummers' method. ${ }^{27} 50 \mathrm{~mL}$ of graphene oxide aqueous solution $\left(3 \mathrm{mg} \mathrm{mL} \mathrm{mL}^{-1}\right.$ ) was ultrasonically mixed for $20 \mathrm{~min}$ to obtain a uniform mixed solution. Then, $150 \mathrm{mg}$ of vitamin $\mathrm{C}$ and different quantities of $\mathrm{MoO}_{3}$ were added and stirred evenly for $30 \mathrm{~min}$ to obtain a uniform sol mixed solution. Then, the mixed solution was divided into 5 small $25 \mathrm{~mL}$ bottles with lids. They were placed in an oven at $95{ }^{\circ} \mathrm{C}$ for $6 \mathrm{~h}$ to obtain $\mathrm{rGO}-\mathrm{MoO}_{3}$ hydrogel, and then soaked in PVA solution for $24 \mathrm{~h}$. Finally, after three times of washing with deionized water, rGO- $\mathrm{MoO}_{3}$ aerogel was obtained through freeze-drying (naming of sample was shown in Table 1).

\subsection{Characterization}

Crushing of samples: in the cold storage, $500 \mathrm{~mL}$ of $\mathrm{rGO}-\mathrm{MoO}_{3}$ aerogel slurry with a concentration of $10 \mathrm{mg} \mathrm{mL} \mathrm{mL}^{-1}$ was rapidy frozen into ice cube with liquid nitrogen. The ice slag was first obtained by crushing it with an ice breaker, and then transferred to the experimental cryogen pulverizer, which added liquid nitrogen in advance for refrigeration. The shredder was opened for $1 \mathrm{~min}$, liquid nitrogen was added for cooling, and sample was continued to grind for $1 \mathrm{~min}$. The powder particle was sieved over 80 mesh, the screened smoothes were put into the tray of the freeze-dryer, and the smoothes were dried at $-5{ }^{\circ} \mathrm{C}$ under the condition of vacuum degree of $10 \mathrm{~Pa}$ for $30 \mathrm{~h}$. After drying, the rGO-MoO${ }_{3}$ aerogel was packed, and the physical and chemical properties was shown in Table 1.

X-ray diffraction (XRD, Rigaku D/max 2500v/pc X-ray diffractometer, $\mathrm{Cu} \mathrm{K} \alpha$ radiation, nickel-filtered, $\lambda=0.15405 \mathrm{~nm})$ test: the measured voltage was $36 \mathrm{kV}$, the current was $20 \mathrm{~mA}$, the scanning speed was $4^{\circ} \min ^{-1}$, and the scanning angle was $5-80^{\circ}$.

Table 1 The properties of $\mathrm{MoO}_{3}, \mathrm{rGO}$ aerogel and $\mathrm{rGO}-\mathrm{MoO}_{3}$ aerogels

\begin{tabular}{|c|c|c|c|c|c|c|c|}
\hline rGO & $1: 0 / 1: 0$ & 326.5 & 84.3 & 16.3 & 310.2 & 2.17 & 97.8 \\
\hline rGO-MoO ${ }_{3}(3: 1)$ & $3: 1 / 3: 1$ & 224.8 & 103.7 & 19.6 & 205.2 & 1.32 & 118.7 \\
\hline rGO-MoO ${ }_{3}(4: 1)$ & $4: 1 / 4: 1$ & 225.1 & 93.3 & 18.4 & 206.7 & 1.51 & 134.5 \\
\hline 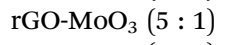 & $5: 1 / 5: 1$ & 236.5 & 92.1 & 22.4 & 214.1 & 1.57 & 144.8 \\
\hline
\end{tabular}

${ }^{a}$ Specific surface area. ${ }^{b}$ Micropore surface area. ${ }^{c}$ Remaining surface area. ${ }^{d}$ Pore volume. ${ }^{e}$ Average pore diameter. 
Fourier transform infrared (FTIR, Bruker Vertex 70 FTIR spectrophotometer) spectra test: $\mathrm{KBr}$ and sample to be tested were placed in an oven at $60{ }^{\circ} \mathrm{C}$ and dried for $2 \mathrm{~h}$, and the FTIR spectra were measured with the wavelength range of $400-4000 \mathrm{~cm}^{-1}$.

Raman (Renishaw INVIA spectrometer) spectra test: the $514.5 \mathrm{~nm}$ radiation from a $20 \mathrm{~mW}$ air-cooled argon ion laser was used as the excitation source.

X-ray photoelectron spectroscopy (XPS, Thermo-VG Scientific) test: the XPS profiles were recorded by an ESCALAB $250 \mathrm{X}$ ray Photoelectron Spectroscopy.

Scanning electron microscopy (SEM, PHILIPS XL-30) test: the surface morphology, element composition and content of sample were observed under the field emission scanning electron microscope with the acceleration voltage of $20.0 \mathrm{kV}$.

Transmission electron microscope (TEM, Dutch electronics Philips Tecnai type 10) test: the sample was ultrasonically dispersed in ethanol solution $\left(0.1 \mathrm{mg} \mathrm{mL}^{-1}\right)$, and 1 to 2 drops of the mixture solution were dropped on carbon film. The morphology and element distribution of sample were observed.

The specific surface area (SSA, Micromeritics 2020 analyzer) test: the specific surface areas of $\mathrm{rGO}$ and $\mathrm{rGO}-\mathrm{MoO}_{3}$ aerogel were detected by automatic specific surface area and pore analyzer. The adsorbent was nitrogen, the degassing mode was heated and vacuum-evacuated, the degassing temperature was $85^{\circ} \mathrm{C}$, the degassing time was $6 \mathrm{~h}$, the ambient temperature was $15{ }^{\circ} \mathrm{C}$, and the saturated vapor pressure was 1.04 bar.

Ultraviolet absorption spectrum (UV/Vis spectra, Hitachi 330 UV-Vis spectrophotometer) test: all the aqueous samples were diluted to $0.05 \mathrm{mg} \mathrm{mL}^{-1}$, and the wavelength range of solid UV/ Vis spectrum was $200-800 \mathrm{~nm}$.

Fluorescence spectrum (Hitachi F4600 fluorescence spectrophotometer) test: all the aqueous samples were diluted to $0.05 \mathrm{mg} \mathrm{mL}^{-1}$. The excitation wavelength of $\mathrm{MoO}_{3}$ was $450 \mathrm{~nm}$, and the excitation wavelength of $\mathrm{rGO}-\mathrm{MoO}_{3}$ aerogel was $250 \mathrm{~nm}$.

The electron paramagnetic resonance (EPR) signals of the samples were recorded at room temperature on Bruker Elexsys E580 EPR spectrometer in X-band ( $m=9.4 \mathrm{GHz}, 3.2 \mathrm{~cm}$ wavelength, X-band).

\subsection{Evaluation of photocatalytic activity}

The visible light photocatalytic activity of rGO- $\mathrm{MoO}_{3}$ aerogel on degrading $\mathrm{MB}$ and $\mathrm{MO}$ was evaluated in the photochemical reactor (XUJIANG XPA-2). A $300 \mathrm{~W}$ xenon lamp containing a $420 \mathrm{~nm}$ filter (HITACHI Y-430) was used, and the reaction system was kept at $25{ }^{\circ} \mathrm{C}$ by circulating cooling water.

$100 \mathrm{mg}$ of $\mathrm{rGO}-\mathrm{MoO}_{3}$ aerogel was put into $200 \mathrm{~mL}$ of $\mathrm{MB}$ solution $\left(40 \mathrm{mg} \mathrm{L}^{-1}\right)$ or MO solution $\left(20 \mathrm{mg} \mathrm{L}^{-1}\right)$, and stirred for $4 \mathrm{~h}$ under dark condition to make the rGO-MoO $\mathrm{M}_{3}$ aerogel and $\mathrm{MB}$ or MO solution reach the adsorption and desorption equilibrium. Then, the whole reaction system was exposed to Xenon light. $4 \mathrm{~mL}$ of solution was taken every $20 \mathrm{~min}$, and MB or MO concentration was determined by a TU-1900 spectrophotometer (MB at $662 \mathrm{~nm}$ or MO at $465 \mathrm{~nm}$ ). The degradation rate $\left(D_{\mathrm{p}}\right)$ was calculated through the formula (1).

$$
D_{\mathrm{p}}=\left[1-\left(C / C_{0}\right)\right] \times 100 \%
$$

where $C_{0}$ was the adsorption equilibrium concentration after dark reaction concentration of $\mathrm{MB}$ or $\mathrm{MO}$ (before visible light reaction), and $C$ was the concentration of $\mathrm{MB}$ or MO solution taken after a fixed visible light reaction time.

\subsection{Stability of photocatalytic properties}

The photocatalytic performance stability of $\mathrm{rGO}-\mathrm{MoO}_{3}$ aerogel was tested by several successive degradation experiments. After each degradation experiment, the $\mathrm{rGO}-\mathrm{MoO}_{3}$ aerogel was taken out and washed with $100 \mathrm{~mL}$ of flowing deionized water for three times without any other treatments. After that, the rGO$\mathrm{MoO}_{3}$ aerogel was freeze-dried for $12 \mathrm{~h}$ at $-55{ }^{\circ} \mathrm{C}$ for the next degradation experiment.

To identify the main reactive species of $\mathrm{rGO}-\mathrm{MoO}_{3}$ aerogel involving in the photodegradation of $\mathrm{MB}$, the trapping experiments were conducted by using EPR spectrometer. In this experiment, isopropyl alcohol (IPA), benzoquinone (BQ) and disodium ethylenediaminetetraacetic acid (EDTA) were used as scavengers and mixed in the aqueous solution to trap the hydroxyl radical $\left(\mathrm{OH}^{\cdot}\right)$, radical anion $\left({ }^{\cdot} \mathrm{O}_{2}{ }^{-}\right)$and hole $\left(\mathrm{h}^{+}\right)$, respectively.

\section{Results and discussion}

\subsection{Composition and structural characterization}

The XRD patterns of GO, rGO, $\mathrm{MoO}_{3}$ and $\mathrm{rGO}-\mathrm{MoO}_{3}$ aerogels were shown in Fig. 1. For GO, there was an obvious corresponding characteristic diffraction peak of (001) crystal plane at $2 \theta=10.30^{\circ}$; for $\mathrm{rGO}$, this corresponding characteristic peak ( $2 \theta$ $=10.30^{\circ}$ ) was not found, but a wide peak occurred at $2 \theta=$ $24.90^{\circ}$, indicating that GO was reduced during the self-assembly of GO sheet into three-dimensional (3D) rGO aerogel. $\mathrm{MoO}_{3}$ had many characteristic peaks at $2 \theta=12.78^{\circ}, 23.32^{\circ}, 25.92^{\circ}, 27.44^{\circ}$, $33.52^{\circ}, 39.05^{\circ}, 45.90^{\circ}$ and $49.70^{\circ}$, corresponding to crystal faces of (020), (110), (040), (021), (111), (060), (200) and (002), respectively. According to the standard card of $\mathrm{MoO}_{3}$ (JCPDS 050508), ${ }^{28}$ the prepared $\mathrm{MoO}_{3}$ was $\alpha-\mathrm{MoO}_{3}$. According to the calculation of Bragg equation, the average layer spacing of (021) crystal plane was $0.32 \mathrm{~nm}$, which was slightly smaller than the theoretical value of $0.33 \mathrm{~nm}$, possibly due to the loss of interlayer water molecule after high temperature treatment. In addition, the average thickness of $\mathrm{MoO}_{3}$ calculated according to Scherer's formula was about $300 \mathrm{~nm} \cdot{ }^{29}$ For $\mathrm{rGO}-\mathrm{MoO}_{3}$ aerogels, the XRD patterns almost coincided with that of rGO aerogel. Except the (021) crystal plane of $\mathrm{MoO}_{3}$, the peaks for $\mathrm{MoO}_{3}$ were not observed in the diffraction patterns of $\mathrm{rGO}-\mathrm{MoO}_{3}$ aerogels. The reason could be ascribed to low diffraction intensity of $\mathrm{MoO}_{3}$ (except the (021) crystal plane). What was more, with the content of $\mathrm{MoO}_{3}$ increased, the intensity of the (021) diffraction peak of $\mathrm{MoO}_{3}$ also gradually increased.

The FTIR spectra of GO, $\mathrm{rGO}, \mathrm{MoO}_{3}$ and $\mathrm{rGO}-\mathrm{MoO}_{3}$ aerogels were shown in Fig. 1. At $3394 \mathrm{~cm}^{-1}$, the elongation vibration of -OH was typical in water, and the corresponding characteristic peaks at $1731 \mathrm{~cm}^{-1}, 1621 \mathrm{~cm}^{-1}, 1384 \mathrm{~cm}^{-1}$ and $1049 \mathrm{~cm}^{-1}$ respectively represented $\mathrm{C}=\mathrm{O}, \mathrm{C}-\mathrm{OH}$, and $\mathrm{C}-\mathrm{O}-\mathrm{C}$ bonds, ${ }^{27}$ indicating that graphite powder was oxidized into GO. In rGO 

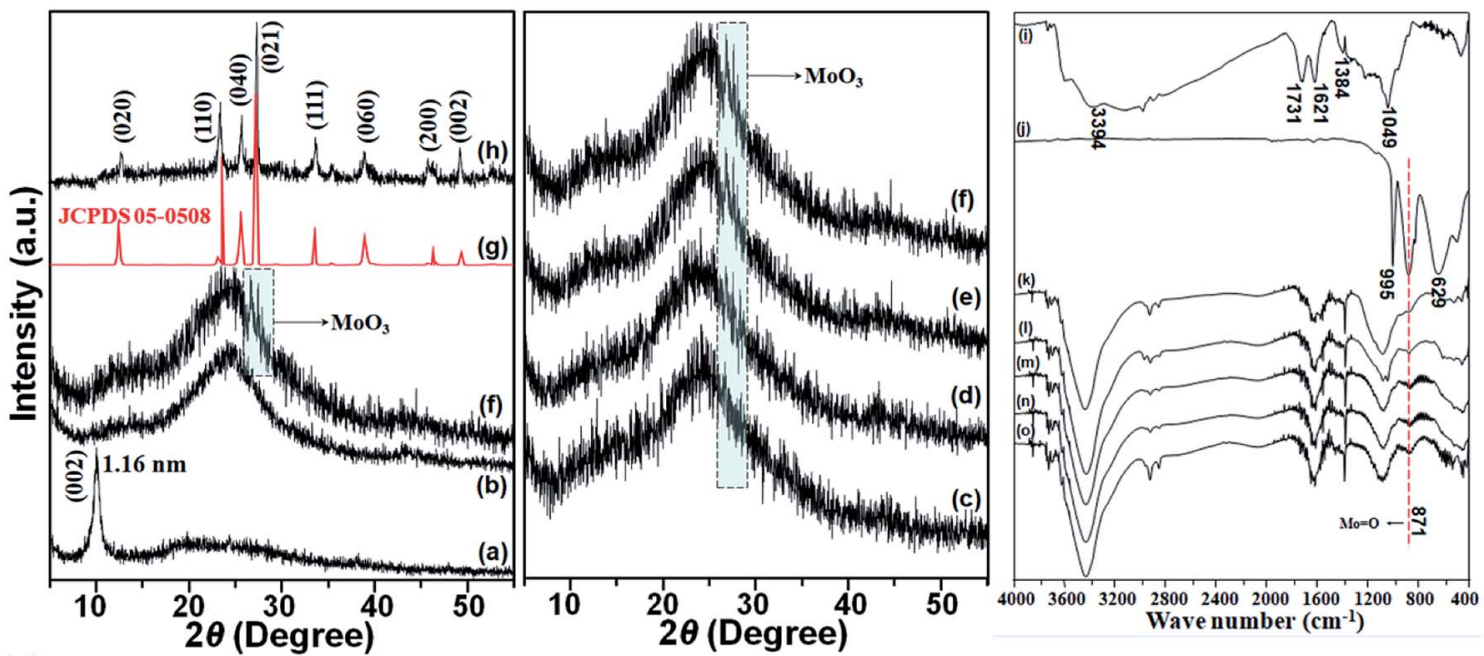

Fig. 1 XRD patterns of (a) GO, (b) rGO, (c) rGO- $\mathrm{MoO}_{3}\left(6: 1\right.$ ), (d) $\mathrm{rGO}-\mathrm{MoO}_{3}$ (5: 1), (e) rGO-MoO 3 (4: 1), (f) rGO-MoO $(3: 1)$, (g) JCPDS $05-0508$, (h) $\mathrm{MoO}_{3}$. FTIR spectra of (i) GO, (j) $\mathrm{MoO}_{3}$, (k) rGO, (l) rGO-MoO $3(6: 1)$, (m) rGO-MoO 3 (5: 1), (n) rGO-MoO $(4: 1)$, (o) rGO-MoO $3(3: 1)$.

and $\mathrm{rGO}-\mathrm{MoO}_{3}$ aerogels, these oxidation functional groups were weak, indicating that GO was reduced to rGO. For $\mathrm{MoO}_{3}$, characteristic peaks at $995 \mathrm{~cm}^{-1}$ and $871 \mathrm{~cm}^{-1}$ were derived from $\mathrm{Mo}=\mathrm{O}$ of $\mathrm{MoO}_{3}$, and characteristic peak at $629 \mathrm{~cm}^{-1}$ corresponded to O-Mo-O bending vibration. ${ }^{30}$ For rGO- $\mathrm{MoO}_{3}$ aerogels, the FTIR spectra almost coincided with that of rGO aerogel. Except the characteristic peak at $871 \mathrm{~cm}^{-1}$ of $\mathrm{MoO}_{3}$, the characteristic peaks for $\mathrm{MoO}_{3}$ were not observed in the characteristic peaks of $\mathrm{rGO}-\mathrm{MoO}_{3}$ aerogels. The reason could be ascribed to the positions of characteristic peaks were almost coincident (except the $871 \mathrm{~cm}^{-1}$ of $\mathrm{MoO}_{3}$ ). What was more, with the content of $\mathrm{MoO}_{3}$ increased, the intensity of characteristic peak at $871 \mathrm{~cm}^{-1}$ of $\mathrm{MoO}_{3}$ also gradually increased.

The Raman and XPS spectra were also performed (Fig. 2). In Fig. 2a, the Raman shifts of the GO at $1354 \mathrm{~cm}^{-1}$ and $1600 \mathrm{~cm}^{-1}$ were respectively the characteristic D- and G-bands with an $I_{\mathrm{D}} / I_{\mathrm{G}}$
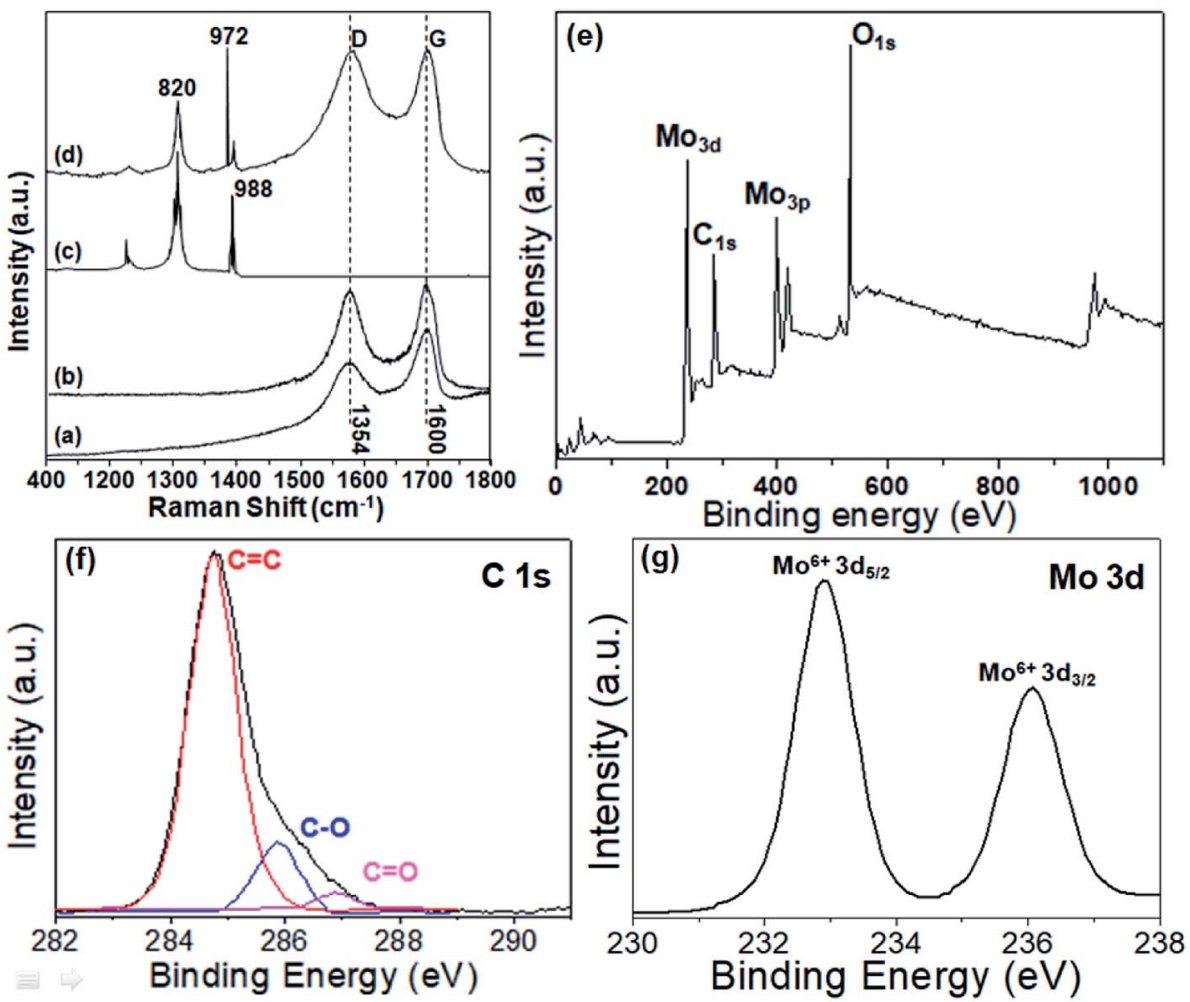

Fig. 2 Raman spectra of (a) GO, (b) $\mathrm{rGO}$, (c) $\mathrm{MoO}_{3}$ and (d) $\mathrm{rGO}-\mathrm{MoO}_{3}$ (3: 1). (e) XPS survey spectrum of rGO-MoO 3 (3: 1). (f) Deconvolution of C 1s high-resolution spectra and (g) $\mathrm{Mo} 3 \mathrm{~d}$ high-resolution spectra of $\mathrm{rGO}-\mathrm{MoO}_{3}(3: 1)$. 
ratio of 0.81 . Moreover, the $I_{\mathrm{D}} / I_{\mathrm{G}}$ ratio of rGO was 1.02 . The $\mathrm{D}$ band represented the defects in the GO lattice of $\mathrm{C}$ atom, and the G-band represented the in-plane stretching vibration of $\mathrm{sp}^{2}$ hybridization of $\mathrm{C}$ atom. For rGO aerogel (Fig. 2b), these bands still existed with an $I_{\mathrm{D}} / I_{\mathrm{G}}$ ratio of 0.97 , and the $I_{\mathrm{D}} / I_{\mathrm{G}}$ ratio increased compared to that of GO. This was due to the formation of smaller and new $\mathrm{sp}^{2}$ domain during reduction. ${ }^{24}$ For $\mathrm{MoO}_{3}$, two main peaks at $820 \mathrm{~cm}^{-1}$ and $988 \mathrm{~cm}^{-1}(\mathrm{M}=\mathrm{O}$ stretching) could be found, respectively, which was attributed to the stretching and bending of Mo-O bonds. ${ }^{14,21}$ In Fig. $2 d$, the Raman shifts of $\mathrm{rGO}-\mathrm{MoO}_{3}(3: 1)$ aerogel combined the Raman shifts of rGO aerogel and $\mathrm{MoO}_{3}$. Besides the peaks of $\alpha-\mathrm{MoO} 3$, the $\mathrm{D}$ and $\mathrm{G}$ peaks of rGO aerogel were also present.

In Fig. 2e, the surface chemistry of the $\operatorname{rGO}^{-M_{0 O}}(3: 1)$ aerogel was displayed, and the $\mathrm{rGO}-\mathrm{MoO}_{3}(3: 1)$ aerogel only contained $\mathrm{C}, \mathrm{O}$ and Mo elements. In Fig. 2f, the individual $\mathrm{C} 1 \mathrm{~s}$

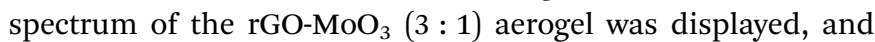
different oxygen-containing functional groups of the $\mathrm{C}=\mathrm{C}$ bond at $284.8 \mathrm{eV}$, the $\mathrm{C}-\mathrm{O}$ bond at $286.2 \mathrm{eV}$ and the $\mathrm{C}=\mathrm{O}$ bond at $288.9 \mathrm{eV}$ were presented. ${ }^{\mathbf{1 4 2 1}}$ In Fig. $2 \mathrm{~g}$, the individual Mo $3 \mathrm{~d}$ spectrum of the rGO- $\mathrm{MoO}_{3}(3: 1)$ aerogel spectrum was displayed. With an integrated peak area ratio of $3: 2$, there were two peaks located at 232.8 and $236.2 \mathrm{eV}$, corresponding to $\mathrm{Mo}(\mathrm{vI})$ for $\mathrm{MoO}_{3}$ in $\mathrm{rGO}-\mathrm{MoO}_{3}(3: 1)$ aerogel. ${ }^{\mathbf{1 4 , 2 1}}$

Nitrogen isothermal adsorption and desorption experiment at $77 \mathrm{~K}$ could further analyze the porous structure of $\mathrm{rGO}-\mathrm{MoO}_{3}$ aerogels, and the results were shown in Table 1 and Fig. S1. $\dagger$ According to IUPAC classification, it could be found that rGO

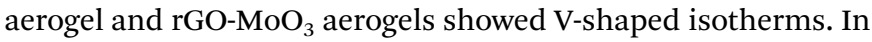
the low and medium pressure section, there was almost no adsorption of all $\mathrm{rGO}-\mathrm{MoO}_{3}$ aerogels, indicating that there was almost no micropore and mesopore in material. At $P / P_{0}>0.9$ (Fig. S1†), rGO aerogel and $\mathrm{rGO}-\mathrm{MoO}_{3}$ aerogels had similar steep and rising peaks and H3-type lag rings, which might be related to macroscopic pores or intercrystalline spaces. Nitrogen isothermal adsorption and desorption curves of rGO$\mathrm{MoO}_{3}$ aerogels with different mass proportions had similar shapes, except that the adsorption capacity would be different at high pressure section due to different pore size.

As shown in Table 1, the specific surface areas of rGO aerogel, rGO- $\mathrm{MoO}_{3}(3: 1)$ aerogel, rGO- $\mathrm{MoO}_{3}(4: 1)$ aerogel, rGO$\mathrm{MoO}_{3}(5: 1)$ aerogel and $\mathrm{rGO}-\mathrm{MoO}_{3}(6: 1)$ aerogel were 326.5, $224.8,225.1,236.5$ and $247.4 \mathrm{~m}^{2} \mathrm{~g}^{-1}$, respectively. The theoretical specific surface area of rGO was over $2000 \mathrm{~m}^{2} \mathrm{~g}^{-1}$, while the specific surface areas of rGO aerogel and $\mathrm{rGO}-\mathrm{MoO}_{3}$ aerogels prepared in this experiment were very low. This was mainly for two reasons: first, the agglomeration and incomplete stripping of rGO aerogel during the self-assembly process of rGO aerogel lamella; second, the rGO and rGO- $\mathrm{MoO}_{3}$ aerogels had to be crushed into powder during the test, which destroyed the pore structure inside the aerogel and affected the specific surface area of the aerogel. The mean pore diameters of rGO aerogel, rGO- $\mathrm{MoO}_{3}(3: 1)$ aerogel, rGO- $\mathrm{MoO}_{3}(4: 1)$ aerogel, rGO- $\mathrm{MoO}_{3}$

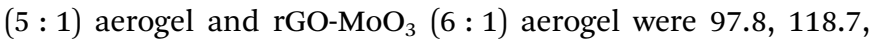
134.5, 144.8 and $132.1 \mathrm{~nm}$, respectively, indicating macroscopic or intergranular pores formed by powder stacking and 3D pores assembled by incomplete lamella.

\subsection{Morphology characterization}

In order to further determine the morphologies of $\mathrm{rGO}-\mathrm{MoO}_{3}$ aerogels, the surface morphologies of them were observed by SEM (Fig. 3). It could be seen from Fig. 3 a that $\mathrm{MoO}_{3}$ had a flake structure with almost uniform size and a thickness of about $300 \mathrm{~nm}$, which was consistent with the XRD calculation. In Fig. 3b, rGO aerogel was 3D layered lamella and stacked structure with surface fold. It could be seen from Fig. $3 \mathrm{c}-\mathrm{f}$ that the $3 \mathrm{D}$
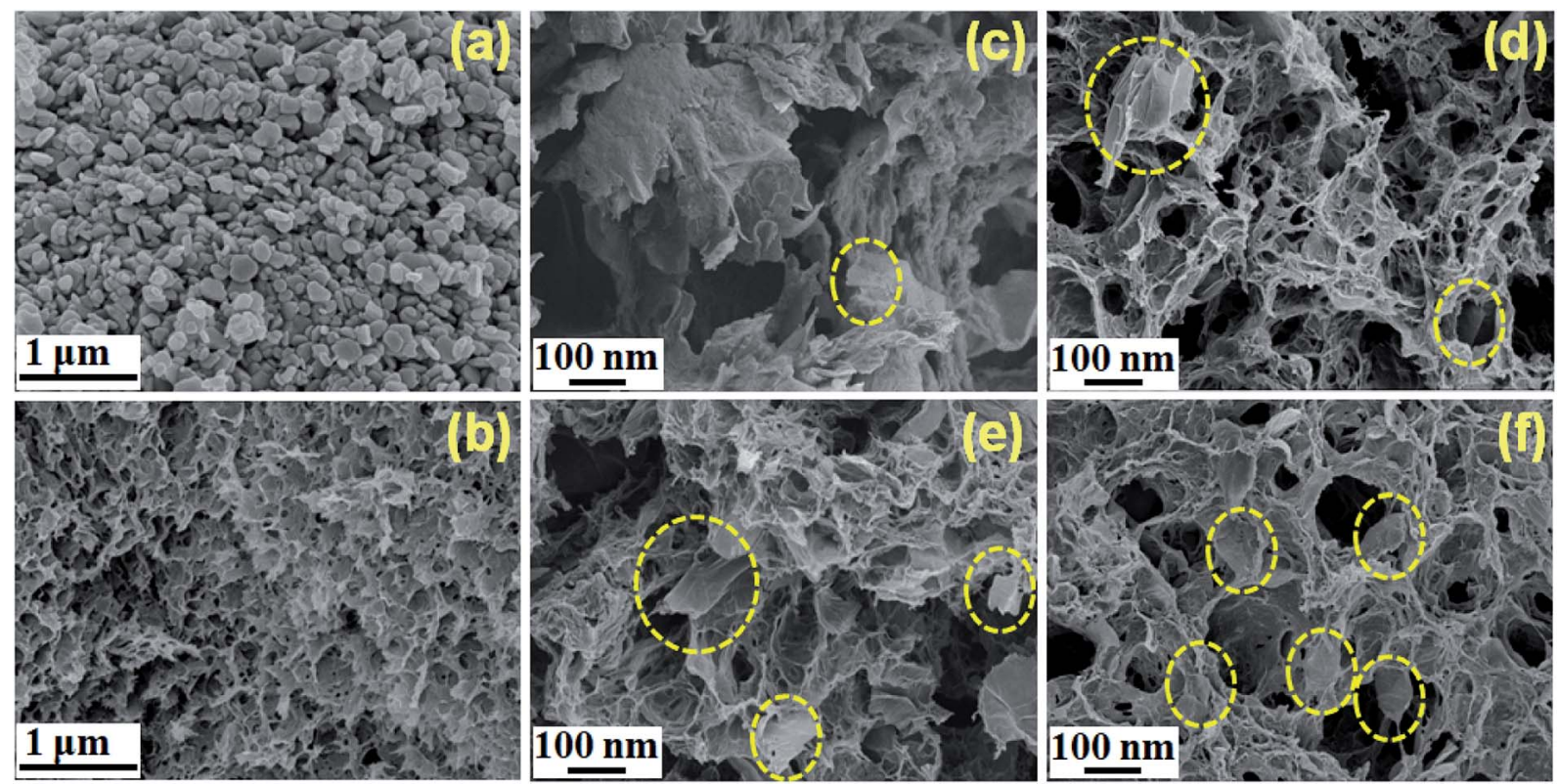

Fig. 3 The SEM images of (a) $\mathrm{MoO}_{3}$, (b) rGO aerogel, (c) rGO- $\mathrm{MoO}_{3}(6: 1)$ aerogel, (d) $\mathrm{rGO}-\mathrm{MoO}_{3}(5: 1)$ aerogel, (e) rGO-MoO 3 (4:1) aerogel, and (f) $\mathrm{rGO}-\mathrm{MoO}_{3}(3: 1)$ aerogel. 
network structure was formed by rGO aerogel during the selfassembly process of rGO lamella. The $\mathrm{rGO}-\mathrm{MoO}_{3}$ aerogel was a porous $3 \mathrm{D}$ mesh structure formed by the interconnection between disordered self-assembled lamella and lamella, and the pores formed were more and messier than those inside rGO aerogel. In addition, we could observe that the $\mathrm{rGO}-\mathrm{MoO}_{3}$ aerogel was composed of two parts. One was the outer wall of aerogel, formed by the accumulation of many rGO sheet layers with rough surface, and another was the core of aerogel. It could be seen that patchy $\mathrm{MoO}_{3}$ was sporadically attached to the surface of rGO sheet layer (yellow dotted circle), indicating that $\mathrm{MoO}_{3}$ was successfully introduced into rGO aerogel.

Fig. 4a was the TEM diagram of $\mathrm{MoO}_{3}$. The test results were completely consistent with those in SEM diagram, and they were uniform sheets. From Fig. 4b, we could see that rGO was a single lamellar layer with slight fold. In the TEM of $\mathrm{rGO}-\mathrm{MoO}_{3}$ aerogels, it could be obviously observed that $\mathrm{MoO}_{3}$ was disorderly scattered on the rGO lamella. In Fig. $4 \mathrm{e}-\mathrm{g}$, the elemental analysis also showed that $\mathrm{rGO}-\mathrm{MoO}_{3}$ aerogel was composed of $\mathrm{C}, \mathrm{O}$ and Mo elements, indicating that $\mathrm{MoO}_{3}$ and rGO were successfully compounded. In addition, the lattice diffraction fringe from $\mathrm{MoO}_{3}$ could be clearly seen in the high-power TEM figure (Fig. 4d). The lattice spacings of $0.322 \mathrm{~nm}$ and $0.398 \mathrm{~nm}$ corresponded to the (021) and (101) diffraction fringes of $\mathrm{MoO}_{3}$, respectively. According to the above characterization results, $\mathrm{MoO}_{3}$ was successfully loaded in rGO aerogel.

Table 1 also showed the actual $\mathrm{MoO}_{3}$ mass ratio in rGO aerogel. The input mass ratio and the actual component mass ratio of $\mathrm{rGO}-\mathrm{MoO}_{3}$ aerogel did not have much difference. Input mass ratios of rGO- $\mathrm{MoO}_{3}$ aerogels were $3: 1,4: 1,5: 1$ and $6: 1$, and the actual component mass ratios were $3: 1,4: 1$,
$5: 1$ and $6: 1$, respectively, which meant that $\mathrm{MoO}_{3}$ was completely loaded on GO.

As could be seen from Fig. 5, after freeze-drying, the volume

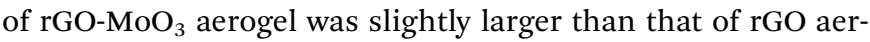
ogel, indicating that the addition of $\mathrm{MoO}_{3}$ increased the volume of rGO aerogel. Putting $100 \mathrm{~g}$ of weight on $\mathrm{rGO}-\mathrm{MoO}_{3}$ aerogels and rGO aerogel, respectively, it could be seen that rGO- $\mathrm{MoO}_{3}$ aerogels would still maintain the intact appearance, while rGO aerogel completely collapsed, which proved that the addition of $\mathrm{MoO}_{3}$ increased the mechanical strength of $\mathrm{rGO}-\mathrm{MoO}_{3}$ aerogels. The $\mathrm{rGO}-\mathrm{MoO}_{3}$ aerogel was placed on kapok fluff, and it was found that kapok fluff did not deform at all, which indicated that $\mathrm{rGO}-\mathrm{MoO}_{3}$ aerogel had the characteristics of low density.

\subsection{Optical properties}

The optical properties of rGO- $\mathrm{MoO}_{3}$ aerogels would directly affect their degradation properties. As shown in Fig. 6a, the absorption range of $\mathrm{MoO}_{3}$ was mainly in the ultraviolet region, and the maximum absorption showed at $440 \mathrm{~nm}$, which was generated by inter-band and exciton transition. By contrast, rGO aerogel had full spectrum absorption. For rGO-MoO $\mathrm{M}_{3}$ aerogels, the absorption range was almost the same with that of rGO aerogel, but the light absorption ability was lower than that of rGO aerogel. What was more, with the content of rGO increased, the light absorption ability of rGO- $\mathrm{MoO}_{3}$ aerogel also gradually increased.

The fluorescence spectrum could reflect the carrier transfer process and the captured process of the semiconductor. It could be seen from Fig. $6 \mathrm{~b}$ that the fluorescence spectrum of rGO$\mathrm{MoO}_{3}$ aerogel showed a certain fluorescence quenching
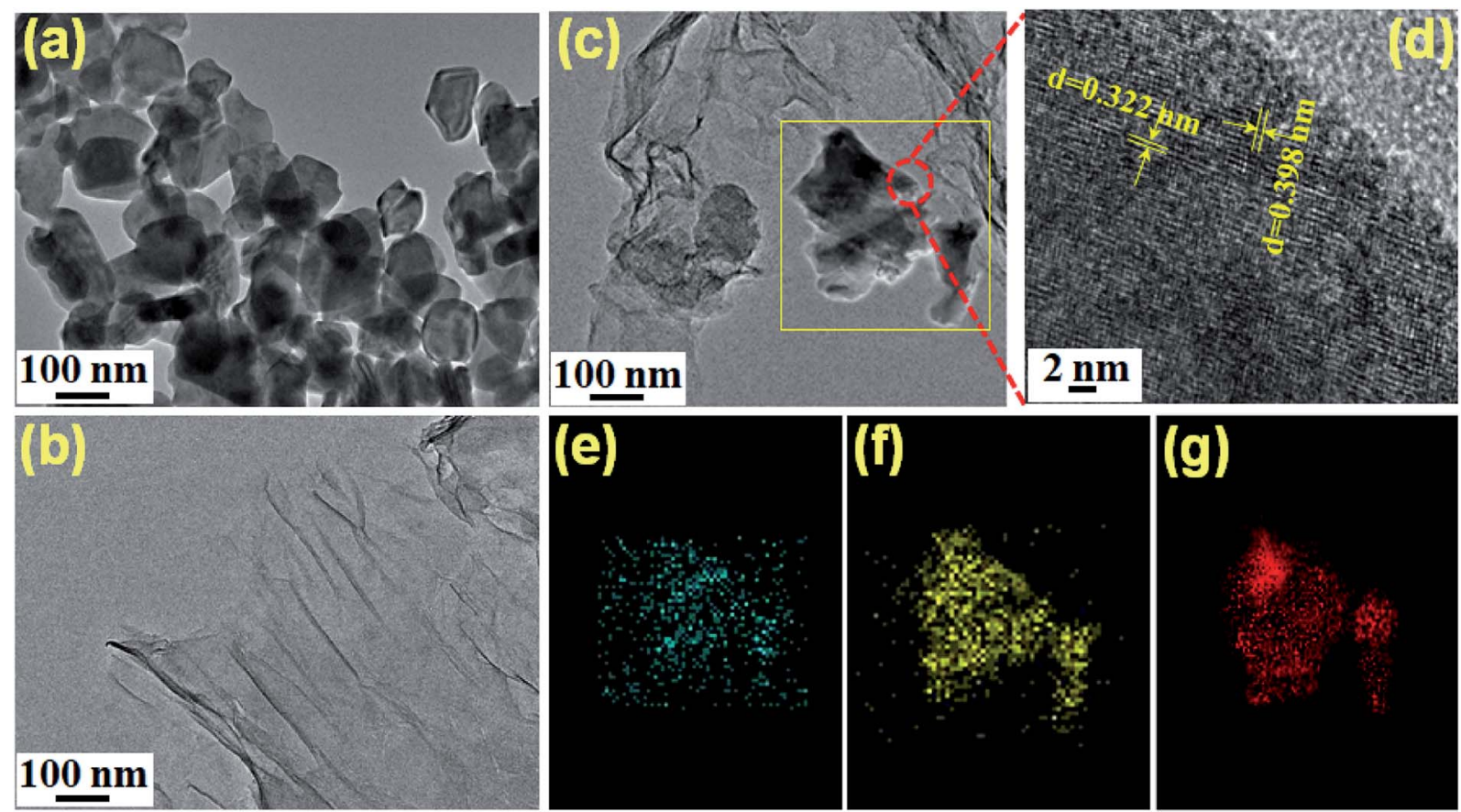

Fig. 4 The TEM images of (a) $\mathrm{MoO}_{3}$, (b) $\mathrm{rGO}$ aerogel, (c) $\mathrm{rGO}-\mathrm{MoO}_{3}$ (3: 1) aerogel, (d) lattice diffraction fringe of $\mathrm{MoO}_{3}$ on $\mathrm{rGO}^{-\mathrm{MoO}_{3}}$ (3: 1 ) aerogel, (e-g) mapping of $\mathrm{MoO}_{3}$ on $\mathrm{rGO}-\mathrm{MoO}_{3}(3: 1)$ aerogel. 

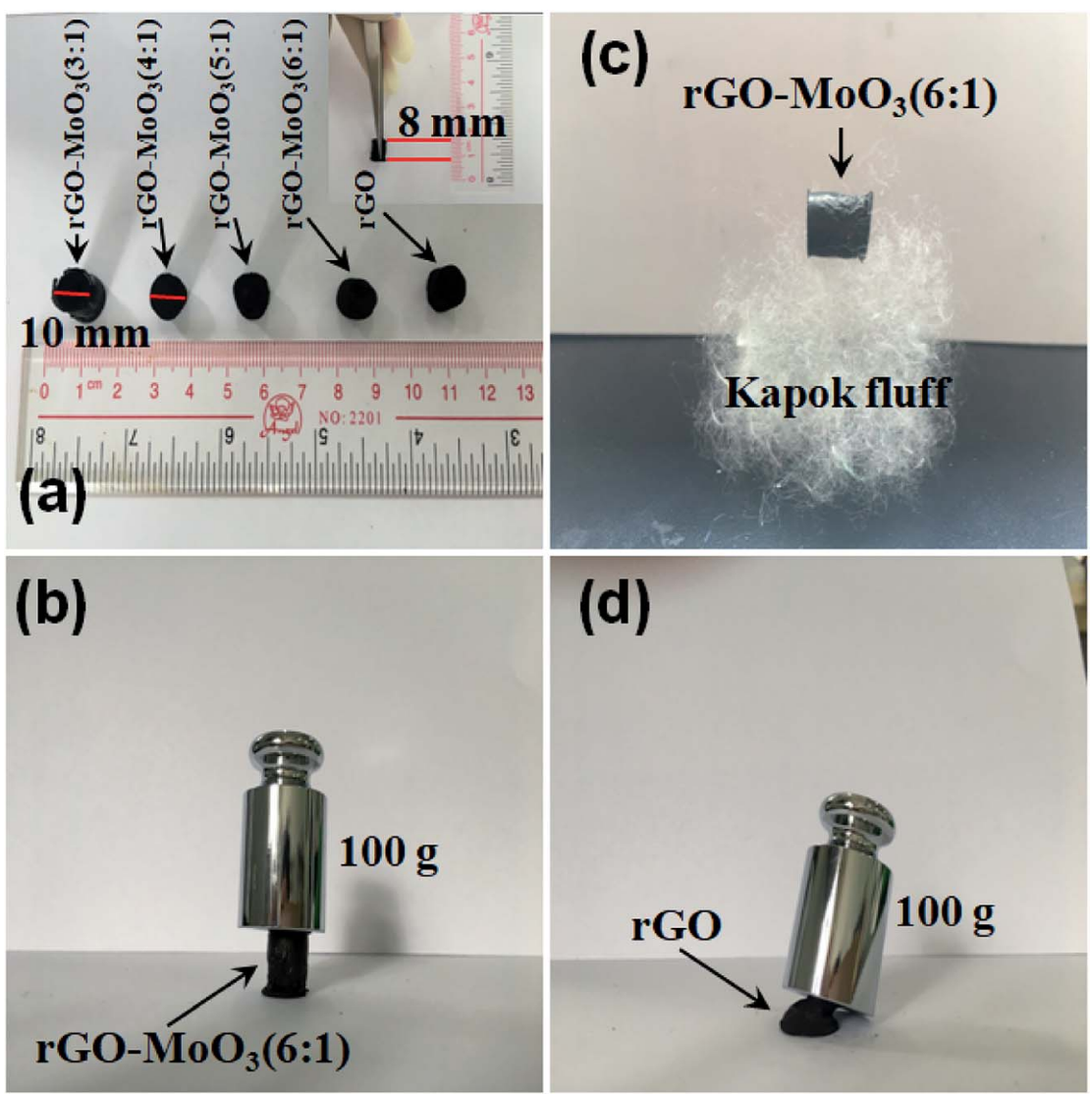

Fig. 5 (a) The digital photos of $\mathrm{rGO}$ and $\mathrm{rGO}-\mathrm{MoO}_{3}$ aerogels; ( $\mathrm{b}$ and c) the mechanical property of $\mathrm{rGO}-\mathrm{MoO}_{3}(6: 1)$ aerogel; (d) the mechanical property of rGO aerogel.

phenomenon. Because nano-flake $\mathrm{MoO}_{3}$ distributed in the interior of $\mathrm{rGO}-\mathrm{MoO}_{3}$ aerogel, the moment when electrons and holes were separated provided convenience for electron conduction and greatly inhibited the recombination of electrons and holes. The 3D structure of $\mathrm{rGO}-\mathrm{MoO}_{3}$ aerogel had a large number of active sites, which increased the effective electron conduction, extended the carrier life, and inhibited the recombination of photo-generated electrons and holes. These excellent properties enhanced the photocatalytic effect of semiconductor material.

\subsection{Dark adsorption and photodegradation performance}

The adsorption performance of $\mathrm{rGO}-\mathrm{MoO}_{3}$ aerogels and rGO aerogel under dark condition was shown in Fig. 7a and b. It could be seen from the dark adsorption experiment of rGO$\mathrm{MoO}_{3}$ aerogels and rGO aerogel that, in the absence of light, the adsorption equilibrium state could be reached between the aerogel and MB solution for about $3 \mathrm{~h}$, or between the aerogel and MO solution for about $2 \mathrm{~h}$. It could be seen that the adsorption performance of $\mathrm{rGO}-\mathrm{MoO}_{3}$ aerogels for $\mathrm{MB}$ and $\mathrm{MO}$
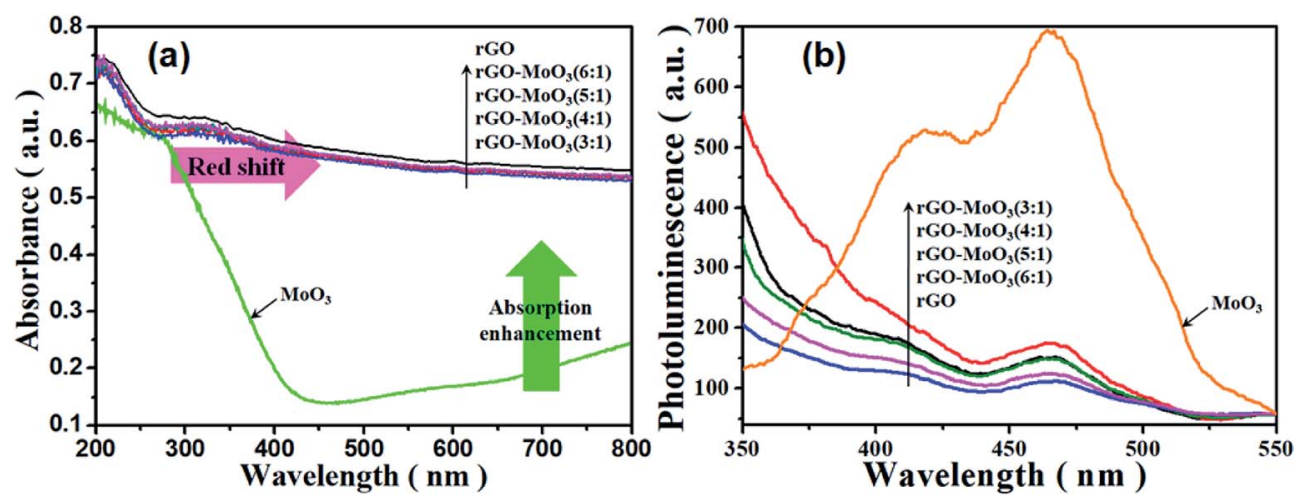

Fig. 6 UV-Vis diffuse reflectance spectra (a) and photoluminescence emission spectra (b) of rGO, $\mathrm{MoO}_{3}, \mathrm{rGO}-\mathrm{MoO}_{3}(3: 1), \mathrm{rGO}-\mathrm{MoO}_{3}(4: 1)$, $\mathrm{rGO}-\mathrm{MoO}_{3}(5: 1)$ and $\mathrm{rGO}-\mathrm{MoO}_{3}(6: 1)$. 

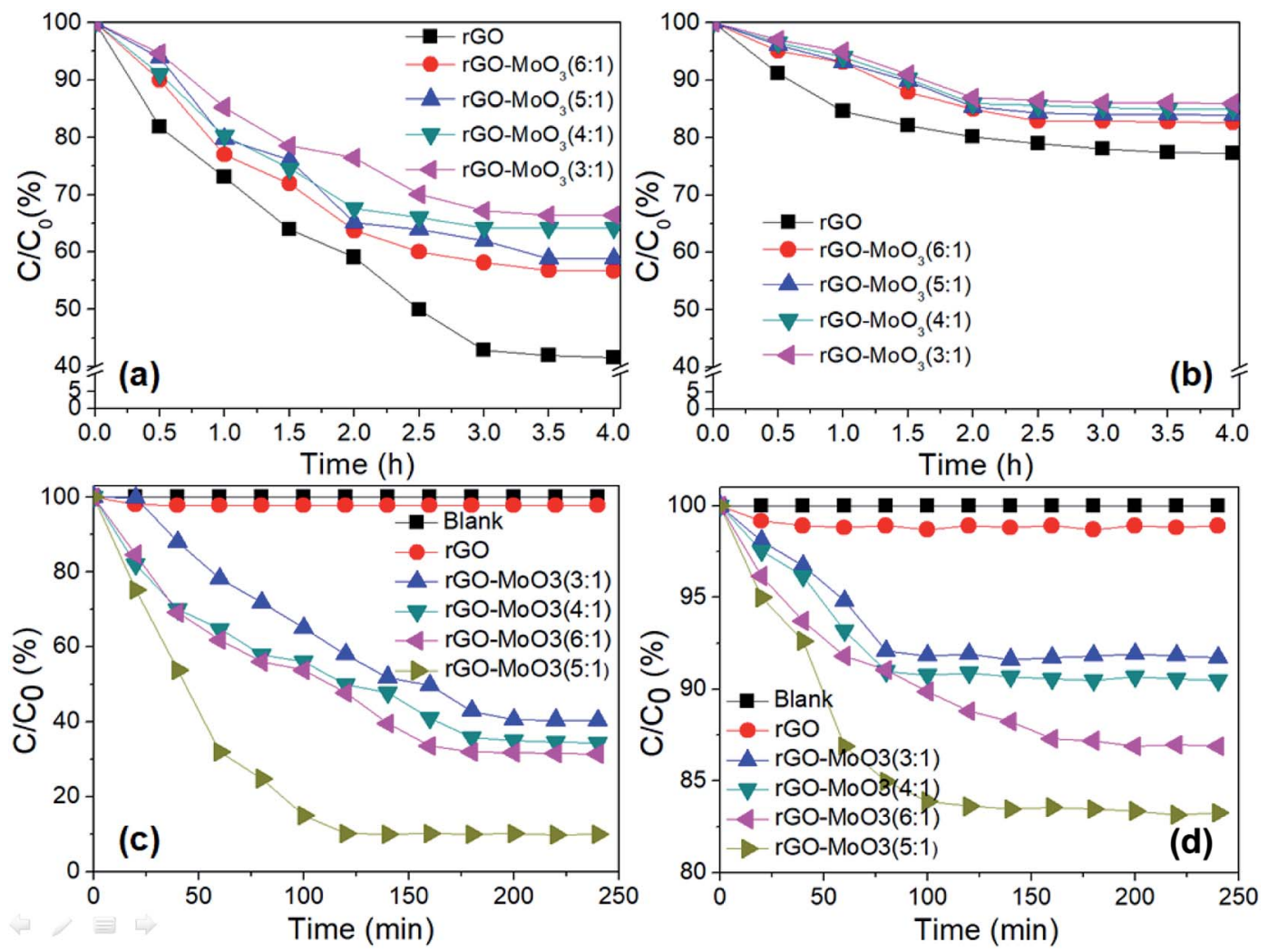

Fig. 7 Absorption performance of $\mathrm{MB}(\mathrm{a})$ and $\mathrm{MO}$ (b) on $\mathrm{rGO}-\mathrm{MoO}_{3}$ aerogels and $\mathrm{rGO}$ aerogel under dark condition. Photodegradation of $\mathrm{MB}$ (c) and $\mathrm{MO}(\mathrm{d})$ under visible light irradiation over $\mathrm{rGO}-\mathrm{MoO}_{3}$ aerogels and $\mathrm{rGO}$ aerogel.

was lower than that of rGO aerogel. This was because $\mathrm{MoO}_{3}$ occupied the adsorption active site on rGO aerogel, and the number of active site greatly affected the adsorption performance of $\mathrm{rGO}-\mathrm{MoO}_{3}$ aerogels on $\mathrm{MB}$ and $\mathrm{MO}$. The adsorption

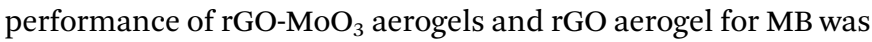
higher than that for MO. This was because MB was a cationic dye. The synergy between dye molecules and $\mathrm{rGO}-\mathrm{MoO}_{3}$ aerogels could promote the adsorption of $\mathrm{MB}$ by $\mathrm{rGO}-\mathrm{MoO}_{3}$ aerogels by virtue of electrostatic adsorption and $\pi-\pi$ conjugate effect. ${ }^{31,32}$ On the contrary, MO was an anion dye, and there was electrostatic repulsion between dye molecules and $\mathrm{rGO}-\mathrm{MoO}_{3}$ aerogels, so rGO- $\mathrm{MoO}_{3}$ aerogels had a stronger adsorption capacity for MB than for MO.

Fig. $7 \mathrm{c}$ and $\mathrm{d}$ showed the photocatalytic diagrams of rGO$\mathrm{MoO}_{3}$ aerogels and rGO aerogel on MB and MO. It could be seen from Fig. $7 \mathrm{c}$ and $\mathrm{d}$ that in the absence of catalyst, the two dyes were very stable under visible light and would not degrade. rGO aerogel had very little photodegradation performance for both $\mathrm{MB}$ and $\mathrm{MO}$ after photoreaction for $240 \mathrm{~min}$. $\mathrm{rGO}-\mathrm{MoO}_{3}$ aerogels hardly degraded $\mathrm{MO}$ in any ratio, and the maximum degradation of $\mathrm{MO}$ by $\mathrm{rGO}-\mathrm{MoO}_{3}(5: 1)$ aerogel was $16.8 \%$. In visible light, the photodegradation performance of $\mathrm{rGO}-\mathrm{MoO}_{3}$ aerogels on $\mathrm{MB}$ was relatively high, and the degradation efficiency of $\mathrm{rGO}-\mathrm{MoO}_{3}(5: 1)$ aerogel on $\mathrm{MB}$ reached $90 \%$. This indicated that $\mathrm{MoO}_{3}$ played a very important role in the photocatalytic efficiency of the whole material. An appropriate amount of $\mathrm{MoO}_{3}$ loading on rGO aerogel could effectively inhibit the recombination of photo-generated electron and hole, so as to improve the photocatalytic performance of rGO$\mathrm{MoO}_{3}$ aerogels.

Under the same condition, the photodegradation efficiency of rGO-MoO${ }_{3}$ aerogels containing different amounts of $\mathrm{MoO}_{3}$ for the two dyes was compared, and it was known that $\mathrm{rGO}-\mathrm{MoO}_{3}$ $(5: 1)$ aerogel had the best degradation rate for both dyes within $240 \mathrm{~min}$, especially for $\mathrm{MB}$, which reached $90 \%$ at $120 \mathrm{~min}$.

\subsection{Recycling performance}

In practical production, in order to be green and economical, the recycling performance of photocatalyst was studied. Due to the poor degradation effect of $\mathrm{rGO}-\mathrm{MoO}_{3}$ aerogels on $\mathrm{MO}$, only the cycling performance of $\mathrm{rGO}-\mathrm{MoO}_{3}(5: 1)$ aerogel on $\mathrm{MB}$ photodegradation was analyzed in this experiment. At the end of each experiment, the catalyst was simply treated with deionized water and then freeze-dried for the next use. In the repeated experiment, the $\mathrm{rGO}^{-\mathrm{MoO}_{3}}(5: 1)$ aerogel could maintain the $3 \mathrm{D}$ structure integrity. The reaction process was suspended in solution with the large-area contact with $\mathrm{MB}$, and the light could be well used (photo in Fig. 8). As shown in Fig. 8, after six cycles of use, the degradation efficiencies of rGO- $\mathrm{MoO}_{3}$ (5 : 1) aerogel to MB were $92.3 \%, 91.9 \%, 91.6 \%, 90.8 \%, 90.7 \%$ and $90.7 \%$, respectively.

$3 \mathrm{D}$ reticular $\mathrm{rGO}-\mathrm{MoO}_{3}$ aerogel could be suspended in reaction solution due to its excellent structure and low density (photo in Fig. 8), so that rGO-MoO ${ }_{3}$ aerogel could fully contact with dye 


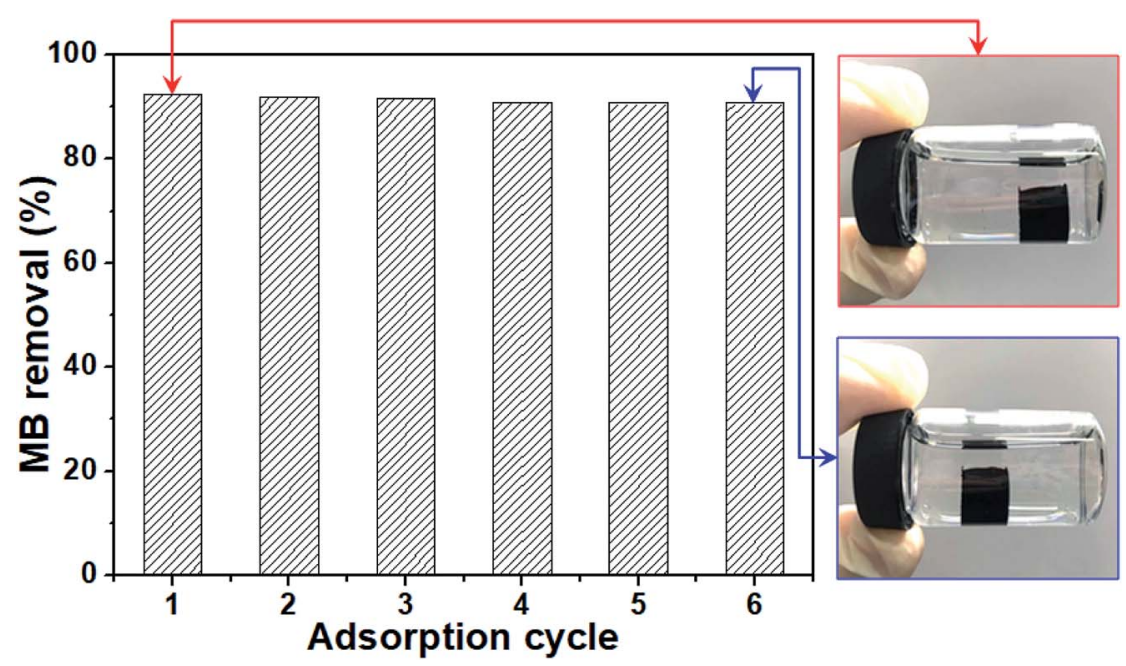

Fig. 8 Cycling run of photocatalytic degradation for $\mathrm{MB}$ using $\mathrm{rGO}-\mathrm{MoO}_{3}(5: 1)$ aerogel.

solution and make full use of visible light source. Moreover, this suspension system also enabled photocatalysis to realize recycling and regeneration in practical application. With high catalytic performance and simple cycle process, $\mathrm{rGO}_{-} \mathrm{MoO}_{3}(5: 1)$ aerogel had a great prospect in practical application.

\subsection{Degradation mechanism}

rGO aerogel could be used as a photocatalyst carrier due to the strong $\pi-\pi$ effect between rGO and metal oxide. ${ }^{33}$ rGO aerogel had a large specific surface area and could provide a large number of active sites. rGO aerogel could act as electron trap and transfer medium. Since $\mathrm{MoO}_{3}$ was a layered structure composed of weak covalent bond connected to each other, these layers could be separated by mechanical action and strong light, thereby generating more photoelectric reaction.

In order to confirm the hypothesis, EPR measurements were used. ${ }^{34-39}$ The EPR spectra of rGO aerogel, $\mathrm{MoO}_{3}$ and rGO-MoO $(5: 1)$ aerogel were shown in Fig. 9a. From Fig. 9a, it was clear that the $\mathrm{rGO}$ aerogel and $\mathrm{MoO}_{3}$ did not show EPR signal while the EPR signal of $\mathrm{rGO}-\mathrm{MoO}_{3}(5: 1)$ aerogel was observed. The

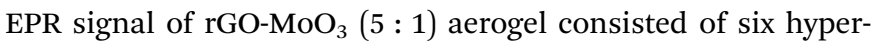
fine lines, suggesting the existence of paramagnetic centers. ${ }^{38,39}$ For $\mathrm{rGO}-\mathrm{MoO}_{3}(5: 1)$ aerogel, the $g$ values corresponding to three distinct anisotropic lines at $g_{1}, g_{2}$ and $g_{3}$ were calculated to be 2.082, 2.013 and 1.951, respectively. ${ }^{38,39}$ The $g_{1}$ and $g_{2}$ might be attributed to the $\mathrm{O}^{-}$as paramagnetic center, and the $g_{3}$ was related to the $\mathrm{Mo}^{5+}$ as paramagnetic center. ${ }^{38,39}$ The presence of $\mathrm{Mo}^{5+}$ in $\mathrm{rGO}-\mathrm{MoO}_{3}(5: 1)$ aerogel indicated the presence of oxygen vacancy.

By performing similar measurements, the reactive species involving in the degradation of dye were determined with adding quenchers (e.g., IPA as $\mathrm{OH}$ scavenger, $\mathrm{BQ}$ as $\mathrm{O}_{2}{ }^{-}$scavenger, and EDTA as $\mathrm{h}^{+}$scavenger). Fig. 9b demonstrated the effects of three quenchers on the photodegradation efficiency of rGO$\mathrm{MoO}_{3}(5: 1)$ aerogel. It was noted that EDTA $\left(\mathrm{h}^{+}\right.$scavenger $)$did not much affect the degradation efficiency of sample. However, the IPA $\left(\mathrm{OH}^{\circ}\right.$ scavenger $)$ and $\mathrm{BQ}\left({ }^{\circ} \mathrm{O}_{2}{ }^{-}\right.$scavenger $)$caused to decrease the degradation efficiency of catalyst. Thus, we
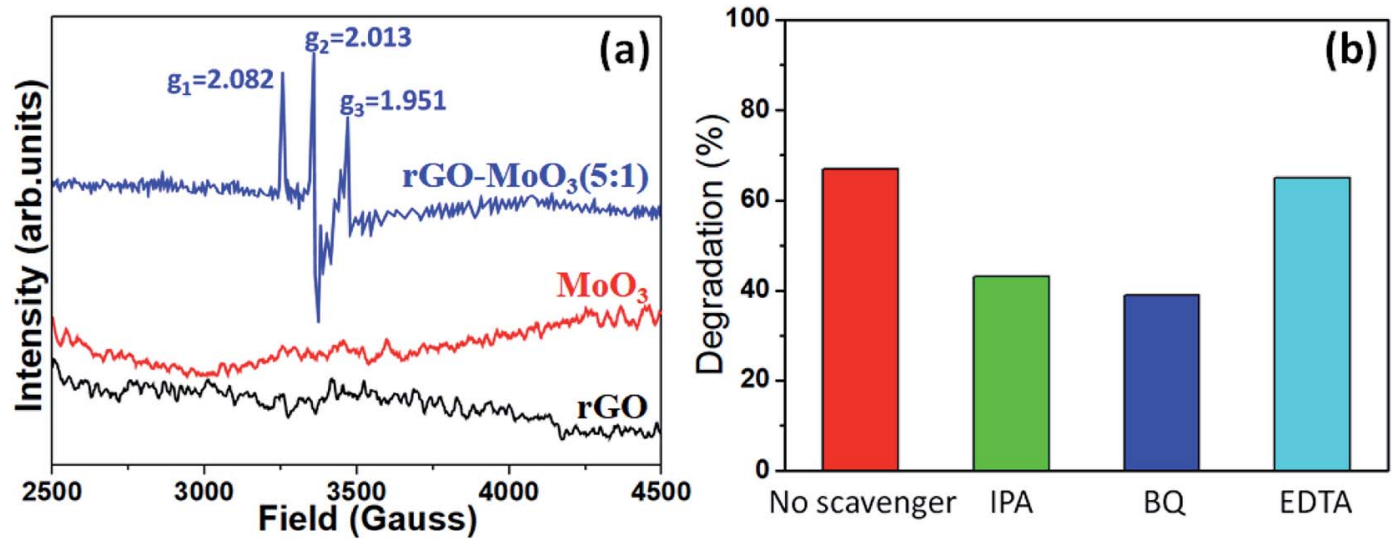

Fig. 9 (a) EPR signals of $\mathrm{rGO}$ aerogel, $\mathrm{MoO}_{3}$ and $\mathrm{rGO}-\mathrm{MoO}_{3}(5: 1)$ aerogel recorded at room temperature. (b) Effects of scavengers (IPA, $\mathrm{BQ}$ and EDTA) on the degradation efficiency of $M B$ dye for 90 min. 
concluded that the $\mathrm{OH}^{\cdot}$ and ${ }^{\circ} \mathrm{O}_{2}{ }^{-}$radicals were the main reactive species involving in the degradation of dye.

In visible light, therefore, the diagram of optoelectronic production and conduction path of $\mathrm{rGO}-\mathrm{MoO}_{3}$ aerogel was shown in Fig. S2. $\dagger$ The similar effects from mesopores and/or a hierarchical porosity for the as-prepared porous material were also reported in other report. ${ }^{40-43}$ Under the excitation of visible light, the $\mathrm{MoO}_{3}$ loading on the rGO lamella produced electrons and holes. rGO capture guided on electronic, and electrons and holes could be effectively separated, thus inhibiting the restructuring of charge carrier and improving the activity of catalyst. . $^{40-43}$

Degradation mechanism could be expressed by the following formula:

$$
\begin{gathered}
\alpha-\mathrm{MoO}_{3}+h \nu \rightarrow \alpha-\mathrm{MoO}_{3}+\mathrm{h}^{+}+\mathrm{e}^{-} \\
\mathrm{h}^{+}+\mathrm{H}_{2} \mathrm{O} \rightarrow \mathrm{OH}^{\cdot}+\mathrm{H}^{+} \text {(oxidation) } \\
\mathrm{e}^{-}+\mathrm{O}_{2} \rightarrow \mathrm{O}_{2}^{-} \text {(reduction) } \\
\mathrm{O}_{2}^{-}+\mathrm{H}^{+} \rightarrow \mathrm{HO}_{2}^{-}+\mathrm{OH}^{-} \\
\mathrm{H}_{2} \mathrm{O}+\mathrm{H}_{2} \mathrm{O} \rightarrow \mathrm{H}_{2} \mathrm{O}_{2}+\mathrm{H}^{+} \rightarrow 2 \mathrm{OH}^{-} \\
\mathrm{O}_{2}^{-}+\mathrm{H}_{2} \mathrm{O} \rightarrow \mathrm{OH}^{-} \\
\mathrm{OH}^{-}+\text {organic molecules } \rightarrow \text { degradation products } \\
\cdot \mathrm{O}_{2}^{-}+\text {organic molecules } \rightarrow \text { degradation products }
\end{gathered}
$$

\section{Conclusions}

The rGO- $\mathrm{MoO}_{3}$ aerogels successfully prepared through chemical reduction assembly mainly contained large pores, almost without micropores or mesoporous. The mean pore diameters of rGO aerogel, rGO- $\mathrm{MoO}_{3}(3: 1)$ aerogel, rGO-MoO $(4: 1)$ aerogel, $\mathrm{rGO}-\mathrm{MoO}_{3}(5: 1)$ aerogel and $\mathrm{rGO}-\mathrm{MoO}_{3}(6: 1)$ aerogel were $97.8,118.7,134.5,144.8$ and $132.1 \mathrm{~nm}$, respectively. The light absorption intensity of $\mathrm{rGO}-\mathrm{MoO}_{3}(6: 1)$ aerogel was higher than those of other $\mathrm{rGO}-\mathrm{MoO}_{3}$ aerogels, and its absorption spectrum had obvious red shift. The presence of rGO increased the absorption spectrum range and active site of the rGO- $\mathrm{MoO}_{3}$ aerogel, thus enhancing its electron conduction, prolonging carrier life and inhibiting electron and hole recombination, and improving the photocatalytic efficiency of the $\mathrm{rGO}-\mathrm{MoO}_{3}$ aerogel. $\mathrm{rGO}-\mathrm{MoO}_{3}$ aerogels had very low density and good mechanical properties, and would not deform under more than 1000 times of its own pressure.

rGO-MoO ${ }_{3}$ aerogels had good adsorption and photocatalytic degradation effect on $\mathrm{MB}$, but the adsorption and degradation effect of them on MO were not obvious. Among them, rGO$\mathrm{MoO}_{3}(5: 1)$ aerogel showed the best degradation effect on MB, displaying high degradation efficiency on MB within 120 min. After six cycles of recycling, the degradation rate of $\mathrm{MB}$ decreased by only $1.6 \%$. The hydroxyl radical $\left(\mathrm{OH}^{\circ}\right)$ and radical anion $\left(\mathrm{O}_{2}{ }^{-}\right)$played an important role in the photodegradation of dye. With high catalytic performance and simple cycle process, $\mathrm{rGO}-\mathrm{MoO}_{3}$ aerogels had a great prospect in practical dye degradation application.

\section{Conflicts of interest}

The authors declare that they have no conflict of interest.

\section{Acknowledgements}

The authors acknowledged financial support from the Foundation of Science and Technology Projects of Guangdong Province (No. 2016A010103047), the Natural Science Foundation of Hunan Province (No. 2019JJ60062), the Science and Technology Innovation Platform Project of Foshan City (2016AG100541, 2017AG100092), the Foshan Technology Research Center (2016GA10161), the Science and Technology Innovation Project of Foshan City (2017AA100161), the Project supported by GDHVPS (2017), the Provincial Key Platform and Major Scientific Research Project of Guangdong College (2017GkQNCX013), the Science and Technology Project of Guangdong Polytechnic (K201713), and the Undergraduate Scientific and Technological Innovation Cultivation Special Fund Project of Guangdong Province (pdjh2019b0721).

\section{References}

$1 \mathrm{M}$. Barlow and T. Clarke, Blue gold: the battle against corporate theft of the world's water, Routledge, 2017.

2 J. Gupta and C. Vegelin, Int. Environ. Agreem.-P., 2016, 16, 433-448.

3 G. Rasul, Environmental Development, 2016, 18, 14-25.

4 A. K. Yadav, S. Jena, B. C. Acharya and B. K. Mishra, Ecol. Eng., 2012, 49, 53-58.

5 A. E. Ghaly, R. Ananthashankar, M. Alhattab and V. V. Ramakrishnan, J. Chem. Eng. Process Technol., 2014, 5, 1-18.

6 N. S. Ngieng, A. Zulkharnain, H. A. Roslan and A. Husaini, ISRN Biotechnol., 2013, 2013, 260730.

7 Y. G. Habba, M. Capochichi-Gnambodoe, L. Serairi and Y. Leprince-Wang, Phys. Status Solidi B, 2016, 253, 14801484.

8 W. P. Low, M. F. M. Din, M. Ponraj, M. A. Fulazzaky, K. Iwao, A. R. Songip and S. Chelliapan, Desalin. Water Treat., 2015, 53, 1342-1351.

9 R. Saravanan, M. M. Khan, V. K. Gupta, E. Mosquera, F. Gracia, V. Narayanan and A. Stephen, J. Colloid Interface Sci., 2015, 452, 126-133.

10 E. Hu and S. Shang, Molecules, 2019, 24, 2755.

11 Z. Naseem, H. N. Bhatti, M. Iqbal, S. Noreen and M. Zahid, Textiles and Clothing: Environmental Concerns and Solutions, 2019, p. 235.

12 M. Wawrzkiewicz, E. Polska-Adach and Z. Hubicki, Sep. Sci. Technol., 2019, 1-15.

$13 \mathrm{~S}$. Hoseinzadeh, R. Ghasemiasl, A. Bahari and A. H. Ramezani, J. Mater. Sci.: Mater. Electron., 2017, 28, 14446-14452. 
14 C. L. Yang, X. W. Zhong, Y. Jiang and Y. Yu, Chin. Chem. Lett., 2017, 28, 2231-2234.

15 X. M. Zhang, K. Z. Li, H. J. Li, J. H. Lu and L. L. Zhang, Nano, 2016, 11, 1650036.

16 R. Giardi, S. Porro, T. Topuria, L. Thompson, C. Fabrizio, C. F. Pirri and H. C. Kim, Appl. Mater. Today, 2015, 1, 27-32.

17 J. Reszczynska, T. Grzyb, J. W. Sobczak, W. Lisowski, M. Gazda, B. Ohtani and A. Zaleska, Appl. Catal., B, 2015, 163, 40-49.

18 A. Barras, M. R. Das, R. R. Devarapalli, M. V. Shelke, S. Cordier, S. Szunerits and R. Boukherroub, Appl. Catal., $B, 2013,130,270-276$.

19 I. Ghaffar, M. F. Warsi, M. Shahid and I. Shakir, Phys. E, 2016, 79, 1-7.

20 L. Y. Huang, H. Xu, R. X. Zhang, X. N. Cheng, J. X. Xia, Y. G. Xu and H. M. Li, Appl. Surf. Sci., 2013, 283, 25-32.

21 M. B. R. Kamalam, S. S. R. Inbanathan and K. Sethuraman, Appl. Surf. Sci., 2018, 449, 685-696.

22 X. C. Zheng, N. Li, S. Jiang, M. Wu and G. P. Zheng, J. Porous Mater., 2019, 26, 733.

23 W. J. Han, C. Zang, Z. Y. Huang, H. Zhang, L. Ren, X. Qi and J. X. Zhong, Int. J. Hydrogen Energy, 2014, 39, 19502-19512. 24 G. Gorgolis and C. Galiotis, 2D Mater., 2017, 4, 032001.

25 Z. W. Tong, D. Yang, J. F. Shi, Y. H. Nan, Y. Y. Sun and Z. Y. Jiang, ACS Appl. Mater. Interfaces, 2015, 7, 25693-25701. 26 Y. Y. Zhang, X. R. Yan, Y. Y. Yan, D. J. Chen, L. H. Huang, J. X. Zhang, Y. Ke and S. Z. Tan, RSC Adv., 2018, 8, 4239-4248. 27 X. Cai, S. Z. Tan, M. S. Lin, A. Xie, W. J. Mai, X. J. Zhang, Z. D. Lin, T. Wu and Y. L. Liu, Langmuir, 2011, 27, 7828-7835.

28 D. P. Debecker, B. Schimmoeller, M. Stoyanova, C. Poleunis, P. Bertrand, U. Rodemerck and E. M. Gaigneaux, J. Catal., 2011, 277, 154-163.

29 R. Q. Tan, Y. He, Y. F. Zhu, B. Q. Xu and L. L. Cao, J. Mater. Sci., 2003, 38, 3973-3978.
30 S. L. Bai, C. Chen, M. Cui, R. X. Luo, A. F. Chen and D. Q. Li, RSC Adv., 2015, 5, 50783-50789.

31 T. Wu, X. Cai, S. Tan, H. Li, J. Liu and W. Yang, Chem. Eng. J., 2011, 173, 144-149.

32 T. Wu, Z. M. Wu, D. Ma, W. B. Xiang, J. L. Zhang, H. D. Liu, Y. L. Deng, S. Z. Tan and X. Cai, Langmuir, 2018, 34, 1518115188.

33 X. Cai, B. Zhang, L. Shi, H. D. Liu, J. L. Zhang, L. H. Huang and S. Z. Tan, Desalin. Water Treat., 2016, 57, 6365-6371.

34 W. W. Zhong, S. J. Shen, M. He, D. Wang, Z. P. Wang, Z. P. Lin, W. G. Tu and J. G. Yu, Appl. Catal., B, 2019, 258, 117967.

35 W. W. Zhong, W. G. Tu, S. S. Feng and A. J. Xu, J. Alloy. Compd., 2019, 772, 669-674.

36 W. W. Zhong, S. J. Shen, S. S. Feng, Z. P. Lin, Z. P. Wang and B. Z. Fang, CrystEngComm, 2018, 20, 7851-7856.

37 W. W. Zhong, Z. P. Lin, S. S. Feng, D. Wang, S. J. Shen, Q. H. Zhang, L. Gu, Z. P. Wang and B. Z. Fang, Nanoscale, 2019, 11, 4407-4413.

38 A. Singh, S. Kumar, B. Ahmed, R. K. Singh and A. K. Ojha, J. Alloys Compd., 2019, 806, 1368-1376.

39 S. Bai, S. Chen, L. Chen, K. Zhang, R. Luo, D. Li and C. C. Liu, Sens. Actuators, B, 2012, 174, 51-58.

40 B. Z. Fang, A. Bonakdarpour, K. Reilly, Y. L. Xing, F. Taghipour and D. P. Wilkinson, ACS Appl. Mater. Interfaces, 2014, 6, 15488-15498.

41 B. Z. Fang, Y. L. Xing, A. Bonakdarpour, S. C. Zhang and D. P. Wilkinson, ACS Sustainable Chem. Eng., 2015, 3, 2381-2388.

42 B. Fang, J. H. Kim, M. S. Kim and J. S. Yu, Acc. Chem. Res., 2013, 46, 1397-1406.

43 J. H. Kim, B. Fang, M. Kim and J. S. Yu, Catal. Today, 2009, 146, 25-30. 